\title{
Vision Dysfunction in Circadian Clock Gene Bmal Mice
}

\author{
Humza Khan, Deepa Mathew, Qianyi Luo, Ashay Bhatwadekar \\ Indiana University School of Medicine, Department of Ophthalmology, Eugene \\ and Marilyn Glick Eye Institute
}

Background and Hypothesis: The circadian rhythm disruption due to shift work results in a range of disorders such as metabolic disturbances, obesity, cardiovascular diseases, and insulin resistance. Interestingly, the core clock gene Brain and Muscle ARNT-Like 1 (Bmal1), is dysfunctional in shift workers. We reasoned dysfunctional Bmal will affect normal vision function. To do so, a genetically modified mouse with disrupted Bmal was assessed for visual function. We hypothesized that Bmal knockout mice will exhibit reduced retinal functions such as impaired acuity, accommodation, and tracking.

Experimental Design or Project Methods: The $\mathrm{Bmal}^{+/-}$mice were inbred and genotyped to obtain wild-types (WT), Bmal ${ }^{+-}$, and $\mathrm{Bmal}^{-{ }_{-}}$. To assess the retinal function, we performed electroretinogram (ERG) recordings at the zeitgeber times (ZT) of $0,6,12$, and 18 which correspond to $7 \mathrm{AM}, 1 \mathrm{PM}, 7 \mathrm{PM}$, and $1 \mathrm{AM}$, respectively, under both scotopic and photopic conditions. The optokinetic response (OKR) assessments were measured in between ZT-3-ZT7.

Results: Consistent with previous studies, the 'a' wave and 'b' wave amplitudes of WT mice demonstrated a circadian rhythm under scotopic condition. There was a decrease in ERG amplitude of $\mathrm{Bmal}^{+-}$, and $\mathrm{Bmal}^{-/-}$when compared to the WT group. Under photopic conditions, the circadian peak of ERG amplitude was reversed for $\mathrm{Bmal}^{-/-}$when compared to both WT and $\mathrm{Bmal}^{+/-}$mice. The OKR assessment was decreased substantially for $\mathrm{Bmal}^{+/-}(0.3748 \mathrm{c} / \mathrm{d})$, and $\mathrm{Bmal}^{-/-}$ $(0.3130 \mathrm{c} / \mathrm{d})$ as compared to the WT mice $(0.4827 \mathrm{c} / \mathrm{d})$.

Conclusion and Potential Impact: Our studies demonstrate that the loss of Bmal leads to vision dysfunction possibly due to impaired rod and cone function. Furthermore, by using a mouse model of circadian rhythm dysfunction, we identified that individuals working on irregular shifts might be vulnerable to vision dysfunction, and our studies warrant timely testing of visual function and strategies for prevention of vision problems in shift workers. 